Revista de Matemática: Teoría y Aplicaciones 2006 13(1) : 17-23

CIMPA - UCR - CCSS ISSN: 1409-2433

\title{
RIGIDEZ DE UNA SUPERFICIE CON PUNTOS SINGULARES
}

\author{
ELENA CHKRYL*
}

Recibido/Received: 25 Ene 2006 - Aceptado/Accepted: 30 Ago 2006

\begin{abstract}
Resumen
Se consideran las deformaciones isométricas infinitesimales de un tipo de superficie con puntos singulares y se demuestra una condición suficiente de rigidez de tales superficies.
\end{abstract}

Palabras clave: Superficie con puntos singulares, fórmula de Gauss, número típico.

\begin{abstract}
The infinitesimal isometric deformations of a type of surface with singular points are considered and a sufficient condition of rigidity of such surfaces is demonstrated.
\end{abstract}

Keywords: Surface with singular points, Gauss formula, typical number.

Mathematics Subject Classification: 32S25, $14 \mathrm{H} 55$.

\section{Introducción}

A la investigación de las deformaciones isométricas infinitesimales de las superficies con puntos singulares (cónicos) representa uno de los problemas suficientemente difícil a resolver, ya que para la investigación de tales superficies no son suficientes los métodos clásicos, incluso para los casos más sencillos para las superficies bidimensionales en el espacio euclídeo tridimensional, se han obtenido solo algunos pocos resultados. En [3] son consideradas las deformaciones isométricas infinitesimales de superficies aristadas de revolución con puntos cónicos, en [8,9] las superficies de revolución con puntos cónicos con algunas condiciones en la variación de las características geométricas a lo largo de las fronteras paralelas; en [1] se investigó el comportamiento del campo de deformaciones y el campo de revolución en un entorno del vértice de un cono convexo.

\footnotetext{
*Escuela de Matemática, Universidad de Costa Rica, 2060 San José, Costa Rica. E-Mail: echkryl@excite.com
} 
En el artículo se consideran las deformaciones isométricas infinitesimales de una superficie cónica $(k+1)$-dimensional con directriz $k$-dimensional y generatrices unidimensionales curvilíneas en el espacio euclídeo $(n+1)$-dimensional, con $1<k<n$, y se establece un criterio de rigidez suficiente de tales superficies.

El artículo esta distribuido de la siguiente manera, en la seccíon 1.1 describimos algunos elementos de la teoría de las superficies $k$-dimensionales en el espacio euclídeo $n$ dimensional, la definición de superficie rígida se da en la seccíon 1.2, en la 1.3 definimos la construcción de una superficie con puntos singulares, el resultado principal del presente trabajo, que trata del criterio de rigidez de una clase de superficies con puntos singulares, con su respectiva demostración se plantea en la seccíon 1.4.

\section{Rigidez de una superficie con puntos singulares}

\subsection{Algunos elementos de la teoría de las superficies $k$-dimensionales en el espacio euclídeo $n$-dimensional}

Sea $E^{n}, \quad n \geq 2$, espacio euclídeo $n$-dimensional, $X$ una $C^{\infty}$-variedad $k$-dimensional, $1<k<n$, de Hausdorff, que satisface el segundo axioma de numerabilidad. Sea $\rho: X \rightarrow E^{n}, C^{2}$-inmersión. Fijando en $E^{n}$ un sistema coordenado cartesiano rectangular, para cada punto $x \in X$, la imagen $\rho(x)$ se considera como el radio vector del punto correspondiente en $E^{n}$ de la superficie $F=\rho(X)$. Para las coordenadas locales $x^{1}, \ldots, x^{k}$ en $X$, a través de $\rho_{i}$ vamos a representar la derivada parcial de $\rho$ con respecto a $x^{i}, \quad i=1 \ldots, k$. En cada punto de $X$, los vectores $\rho_{1}, \ldots, \rho_{k}$ forman una base del plano tangente de la superficie $F$ en el punto correspondiente. A través de $\nu_{1}, \ldots, \nu_{p}, \quad p=1, \ldots, n-k$, representemos campos vectoriales clase $C^{1}$ en la variedad $X$ con los valores en $E^{n}$ que forman en cada punto una base ortonormal del plano normal de la superficie $F$ en $E^{n}$.

En cada punto de la variedad $X$ los vectores $\rho_{1}, \ldots, \rho_{k}, \nu_{1}, \ldots, \nu_{p}$ representan una base del espacio $E^{n}$. La descomposición de las derivadas $\rho_{i j}=\frac{\partial^{2} \rho}{\partial x^{i} \partial x^{j}}, i, j=1, \ldots, k$, por esta base, conduce a las fórmulas de Gauss

$$
\rho_{i j}=\sum_{\ell=1}^{k} \Gamma_{i j}^{\ell} \rho_{\ell}+\sum_{\sigma=1}^{p} b_{i j}^{\sigma} \nu_{\sigma},
$$

donde $\Gamma_{i j}^{\ell}=\Gamma_{j i}^{\ell}$ son los símbolos de Christoffel de la superficie $F, b_{i j}^{\sigma}=b_{j i}^{\sigma}$ son los coeficientes de la segunda forma fundamental de $F$ con respecto al vector normal $\nu_{\sigma}$, $i, j, \ell=1, \ldots, k, \sigma=1, \ldots, n-k$ ver $[4]$.

\subsection{Deformaciones infinitesimales isométricas de superficies multidimen- sionales}

Consideremos una deformación infinitesimal isométrica de una superficie regular $F$. Sea $\delta r$ el campo de deformaciones isométricas correspondiente. Los campos vectoriales $r$ y $\delta r$ 
satisfacen la ecuación

$$
d r \cdot d \delta r=0 .
$$

El campo de deformaciones isométricas y la deformación isométrica infinitesimal correspondiente de $F$, se llaman triviales si $\delta r$ tiene la forma

$$
\delta r=\Omega r+\omega
$$

donde $\Omega$ es un bivector constante de $\wedge^{2} E^{n}$, $\omega$ es un vector constante de $E^{n}, \Omega r$ es el producto interno de un bivector por un vector. La superficie $F$ se llama rígida si todo campo de deformaciones isométricas es trivial.

\subsection{Construción de una superficie con puntos singulares}

Sea $E^{n+1}, n \geq 2$, espacio euclídeo $(n+1)$-dimensional, $E^{n}$ un plano $n$-dimensional en $E^{n+1}, X$ una $C^{\infty}$-variedad $k$-dimensional, $1<k<n$, de Hausdorf, que satisface el segundo axioma de numerabilidad. El plano $E^{n}$ vamos a considerar como el espacio euclídeo $n$ dimensional con estructura euclídea inducida desde $E^{n}$. Vamos a suponer que el origen $O$ del sistema coordenado de $E^{n+1}$ no pertenece al plano $E^{n}$. Considerando el plano real $\mathbb{R}^{2}$ como una variedad bidimensional, definimos $C^{2}$-aplicación $r: X \times \mathbb{R}^{2} \rightarrow E^{n+1}$ por medio de la fórmula

$$
r=x^{k+1} \rho\left(x^{1}, \ldots, x^{k}\right)+x^{k+2} n,
$$

donde $\left(x^{k+1}, x^{k+2}\right) \in \mathbb{R}^{2} x^{1}, \ldots, x^{k}$ son coordenadas locales en $X, n$ vector unitario en $E^{n+1}$, ortogonal a $E^{n}$. Representemos a través de $S$ la imagen $r\left(X \times \mathbb{R}^{2}\right) \subset E^{n+1}$. Notemos que sobre la subvariedad definida por la ecuación $x^{k+1}=0$ de la variedad $X \times \mathbb{R}^{2}$, la aplicación $r$ no es inmersión. A esta subvariedad le corresponde una recta en la superficie $S$.

\subsection{Criterio de rigidez de una clase de superficies con puntos singulares}

Para la superficie $S$ es válido el teorema:

Teorema 1 Si la superficie $F$ es rígida en el espacio euclídeo n-dimensional y en cada punto el número típico $t$ satisface $t \geq 2$, entonces la superficie $S$ es rígida en $E^{n+1}$ con respecto a las deformaciones isométricas infinitesimales.

Demostración: En [2] se demostró que si la superficie $F$ es rígida en $E^{n}$ y en cada punto posee número típico $t \geq 2$, entonces la superficie $\tilde{S}$ definida por la ecuación $\tilde{r}=x^{k+1} \rho$, es rígida con respecto a las deformaciones isométricas infinitesimales en $E^{n+1}$ y todo su campo de deformaciones isométricas en $E^{n+1}$ es trivial:

$$
\delta \tilde{r}=\Omega \tilde{r}+\omega
$$

donde $\Omega$ es un bivector constante de $\wedge^{2} E^{n+1}, \omega$ es un vector constante en $E^{n+1}$. 
Usando en (1) derivadas parciales en coordenadas locales $x^{1}, \ldots, x^{k}, x^{k+1}, x^{k+2}$ en $X \times \mathbb{R}^{2}$, podemos escribir en la forma siguiente el sistema de deformaciones isométricas infinitesimales de la superficie $S$ :

$$
\begin{gathered}
x^{k+1} \rho_{i} \cdot \delta r_{j}+x^{k+1} \rho_{j} \cdot \delta r_{i}=0, \quad i, j=1, \ldots, k \\
x^{k+1} \rho_{i} \cdot \delta r_{k+1}+\delta r_{i} \cdot \rho=0, \quad i=1, \ldots, k \\
x^{k+1} \delta r_{k+2} \cdot \rho_{i}+\delta \rho_{i} \cdot n=0, \quad i=1, \ldots, k, \\
\delta r_{k+1} \cdot \rho=0, \\
\delta r_{k+2} \cdot n=0, \\
\delta r_{k+2} \cdot \rho+\delta r_{k+1} \cdot n=0,
\end{gathered}
$$

donde "." significa el producto escalar en $E^{n+1}$. Observemos que el sistema (4) coincide con el sistema de deformaciones isométricas infinitesimales de la superficie $\tilde{S}$. Luego, toda solución de este sistema se escribe como:

$$
\delta r=x^{k+1} \Omega\left(x^{k+2}\right) \rho+\omega\left(x^{k+2}\right),
$$

donde $\Omega\left(x^{k+2}\right), \omega\left(x^{k+2}\right)$ no dependen de $x^{1}, \ldots, x^{k}, x^{k+1}, \Omega \rho$ representa el producto interno de un bivector por un vector.

Las derivadas de $\delta r$ con respecto a $x^{i}, i=1, \ldots, k, x^{k+1}, x^{k+2}$ adquieren la siguiente forma:

$$
\begin{aligned}
\delta r_{i} & =x^{k+1} \Omega \rho_{i}, \\
\delta r_{k+1} & =\Omega \rho, \\
\delta r_{k+2} & =x^{k+2} \Omega_{k+2} \rho+\omega_{k+2} .
\end{aligned}
$$

Sustituyendo estas expresiones en (4), (5), (6), (7), (8), (9), notemos que (4) y (6) se convierten en identidades y para (5), (7), (8), (9) obtenemos:

$$
\begin{gathered}
\Omega\left[\rho_{i}, n\right]+x^{k+1} \Omega_{k+2}\left[\rho, \rho_{i}\right]+\omega_{k+2} \cdot \rho_{i}=0, \\
x^{k+1} \Omega_{k+2}[\rho, n]+\omega_{k+2} \cdot n=0, \\
\Omega[\rho, n]+\omega_{k+2} \cdot \rho=0,
\end{gathered}
$$

donde $[*, *]$ significa el producto externo de vectores en $E^{n+1}, \Omega[\rho, n]$ significa el producto escalar de bivectores.

Derivando la igualdad (12) con respecto a $x^{i}, i=1, \ldots, k$, y sustituyendo en (10), esta última ecuación la podemos escribir como

$$
\Omega_{k+2}\left[\rho, \rho_{i}\right]=0 .
$$

La derivación de esta igualdad con respecto a $x^{j}, j=1, \ldots, k$, nos da:

$$
\Omega_{k+2}\left[\rho_{j}, \rho_{i}\right]+\Omega_{k+2}\left[\rho, \rho_{i j}\right]=0 .
$$


Alternando aquí los subíndices $i, j$ y sumando la ecuación (14) con el resultado obtenido, podemos establecer que

$$
\Omega_{k+2}\left[\rho, \rho_{i j}\right]=0 .
$$

Utilizando las fórmulas de Gauss, de lo anterior se obtiene

$$
\Omega_{k+2}\left[\rho, \nu_{\sigma}\right]=0, \quad \sigma=1, \ldots, n-k .
$$

Derivamos ahora la ecuación (11) con respecto a $x^{k+1}$ :

$$
\Omega_{k+2}[\rho, n]=0 .
$$

De (13), (15), (16) se tiene que el vector $\Omega_{k+2} \rho$ es ortogonal a los vectores $\rho_{i} \nu_{\sigma}, n$ en $E^{n+1}$. Por lo tanto

$$
\Omega_{k+2} \rho=0 .
$$

De aquí, para $\delta r_{k+2}$ tenemos $\delta r_{k+2}=\omega_{k+2}$ y el sistema de deformaciones isométricas infinitesimales lo podemos escribir en la forma:

$$
\begin{gathered}
\Omega\left[\rho_{i}, n\right]+\omega_{k+2} \cdot \rho_{i}=0, \\
\Omega[\rho, n]+\omega_{k+2} \cdot \rho=0, \\
\omega_{k+2} \cdot n=0 .
\end{gathered}
$$

Derivando la igualdad (19) con respecto a $x^{k+2}$ y usando (16), obtenemos

$$
\omega_{k+2} k+2 \cdot \rho=0
$$

Derivamos la última ecuación primero con respecto a $x^{i}, \quad i=1, \ldots, k$, luego con respecto a $x^{j}, \quad i=1, \ldots, k$. Los resultados de estas operaciones son:

$$
\begin{gathered}
\omega_{k+2 k+2} \cdot \rho_{i}=0, \\
\omega_{k+2 k+2} \cdot \rho_{i j}=0 .
\end{gathered}
$$

Aplicando en (22) las fórmulas de Gauss, encontramos

$$
\omega_{k+2 k+2} \cdot \nu_{\sigma}=0, \quad \sigma=1, \ldots, n-k .
$$

De (20), (21), (23) podemos establecer que

$$
\omega_{k+2 k+2}=0 .
$$

Integrando, obtenemos:

$$
\begin{gathered}
\omega_{k+2}=c, \quad c=\text { const } \\
\omega=x^{k+2} c+\tilde{c}, \quad \tilde{c}=\text { const } .
\end{gathered}
$$

Luego, para $\delta r$ tenemos

$$
\delta r=x^{k+1} \Omega \rho+x^{k+2} c+\tilde{c} .
$$


Ahora, la igualdad (19) la podemos escribir como $\Omega[\rho, n]+c \cdot \rho=0$ o $(-\Omega n+c) \cdot \rho=0$. Derivamos con respecto a $x^{i}, \quad i=1, \ldots, k$

$$
(-\Omega n+c) \cdot \rho_{i}=0
$$

Derivando esta ecuación con respecto a $x^{j}, j=1, \ldots, k$ y utilizando las fórmulas de Gauss, obtenemos:

$$
(-\Omega n+c) \cdot \nu_{\sigma}=0, \quad \sigma=1, \ldots, n-k .
$$

Tomando en consideración que $\omega_{k+2}=c$ y la igualdad (20) hallamos:

$$
(-\Omega n+c) n=0 .
$$

Entonces $c=\Omega n \mathrm{y}$

$$
\delta r=x^{k+1} \Omega \rho+x^{k+2} \Omega n+\tilde{c} .
$$

Falta demostrar que $\Omega=$ const. En vista que $\Omega n=c$, donde $c=$ const, se obtiene $\Omega_{k+2} n=0$. Multiplicamos esta igualdad por $\nu_{\sigma}, \quad \sigma=1, \ldots, n-k$ :

$$
\Omega_{k+2}\left[n, \nu_{\sigma}\right]=0,
$$

ahora por $\rho_{i}, \quad i=1, \ldots, k$ :

$$
\Omega_{k+2}\left[n, \rho_{i}\right]=0 .
$$

La derivación de $(17)$ con respecto a $x^{i}$ y $x^{j}, i, j=1, \ldots, k$, y la aplicación de las fórmulas de Gauss conduce a

$$
b_{i j}^{\sigma} \Omega_{k+2} \nu_{\sigma}=0 .
$$

Multiplicamos por $\nu_{\tau}, \quad \tau=1, \ldots, n-k$ :

$$
b_{i j}^{\sigma} \Omega_{k+2}\left[\nu_{\sigma}, \nu_{\tau}\right]=0 .
$$

De aquí hallamos:

$$
\Omega_{k+2}\left[\nu_{\sigma}, \nu_{\tau}\right]=0 .
$$

Derivando (16) con respecto a $x^{i}, \quad i=1, \ldots, k$, multiplicando la igualdad obtenida primero por $\rho_{j}, \quad j=1, \ldots, k$ y después por $\nu_{\sigma}, \quad \sigma=1, \ldots, n-k$, obtenemos:

$$
\begin{aligned}
& \Omega_{k+2}\left[\rho_{i}, \rho_{j}\right]=0 . \\
& \Omega_{k+2}\left[\rho_{i}, \nu_{\sigma}\right]=0 .
\end{aligned}
$$

De acuerdo con (25), (26), (27), (28), (29) se tiene que $\Omega_{k+2}=0$ y por consiguiente

$$
\Omega=\text { const. }
$$

El teorema queda demostrado.

En [5] se demostró que toda superficie con el número típico $t \geq 3$, es rígida en el espacio correspondiente. De acuerdo con este resultado se establece.

Corolario 1 Si la superficie $F$ en cada punto posee el número típico $t \geq 3$, entonces la superficie $S$ es rígida en $E^{n+1}$. 


\section{Referencias}

[1] Boyarskii, B.V.; Vekua, I.N. (1958) "Demostración de la rigidez de las superfices convexas a trozos regulares cerradas de curvatura no negativa", Izvestia AC URSS. Serie mat. 22(2): 165-176.

[2] Chkryl, E. (2000) "Rigidez de un cono multidimensional", Izv. Vuzov. SeveroKavkazskii región 4: 20-22.

[3] Con-Vossen, S. E. (1959) Algunos Resultados de la Geometría Diferencial Total. M.

[4] Eyzenkhart, L. P. (1948) Geometría de Reimann. M. 316 p.

[5] Markov, P.E. (1992) "Sobre la inmersión de las métricas, las cuales están casi inmersas", Ucr. Geom. Sbornik. 35: 49-67.

[6] Markov,P.E. (1997) "Deformaciones isométricas infinitesimales y analíticas generales de una inmersión 1", Izv. Vusov. Matemática 9: 21-34.

[7] Markov, P.E. (1997) "Deformaciones isométricas infinitesimales y analíticas generales de una inmersión 2", Izv. Vusov. Matemática 9: 41-51.

[8] Mijailov, L.G.; Usmanov, Z.D. (1965) "Investigación de la teoría de funciones y ecuaciones diferenciales por condiciones de frontera", Dushambe: 21-53.

[9] Mijailov, L.G.; Usmanov, Z.D. (1966) "Exposición sobre deformaciones isométricas infinitesimales de superficies de revolución de curvatura positiva con puntos cónicos o parabólicos en el polo", Academia de Ciencias de la URSS 166(4): 791-794. 